\title{
ENCAPSULACIÓN DE PROBIÓTICOS PARA APLICACIONES ALIMENTICIAS
}

\author{
Yeimy Alejandra Rodríguez R. ${ }^{1}$ \\ Andrés Felipe Rojas G. ${ }^{2}$ \\ Sneyder Rodríguez B. ${ }^{3}$
}

\section{RESUMEN}

Losalimentosfuncionales contienencomponentes activos que con un consumo habitual favorecen la salud de consumidor. Dentro del concepto de funcional se encuentran los alimentos con microorganismos probióticos, los cuales al ser ingeridos en dosis adecuadas confieren diversos beneficios, estos microorganismos son sensibles a factores tecnológicos y ambientales que pueden reducir su viabilidad, estabilidad y su capacidad funcional. Existen tecnologías como la encapsulación, las cuales permiten mejorar la estabilidad de los probióticos al protegerlos mediante un material de recubrimiento. En este trabajo se realizó una búsqueda sistemática en diversas bases de datos sobre los probióticos más empleados en la industria de alimentos, su capacidad catalítica en matrices alimenticias, métodos de encapsulación, tipos de matrices en la encapsulación, estabilidad bajo condiciones gastrointestinales y los mecanismos de liberación. Se encontró que la encapsulación, además de favorecer la estabilidad de los microorganismos probióticos frente a factores adversos, condiciona según sus características, su aplicación e incorporación en matrices alimenticias de diversas cualidades.

Palabras clave: alimentos funcionales, probiótico, matriz de encapsulación, actividad catalítica, liberación de probiótico.

\section{ENCAPSULATION OF PROBIOTICS FOR FOOD APPLICATIONS}

\section{ABSTRACT}

Functional foods contain active components, which under their regular use favor consumers' health. Within the functional concept food with probiotics are found, which when ingested in adequate doses confer various benefits. These microorganisms are sensitive to technological and environmental factors that can reduce their viability, stability, and functional capacity. There are some technologies such as encapsulation, which allow improving the stability of probiotics by protecting them with a coating material.

\footnotetext{
${ }^{1}$ Ingeniera Química, Magíster en Ingeniería-Ingeniería Química, Facultad de Ingeniería y Arquitectura, Laboratorio de Alimentos, Universidad Nacional de Colombia, Manizales, Colombia. Correo electrónico: yearodriguezre@unal.edu.co. 2 Ingeniero Químico, Ph.D. Profesor Asociado en Dedicación Exclusiva. Director del Grupo de Investigación en Aprovechamiento de Residuos - GIAR, Facultad de Ingeniería y Arquitectura, Universidad Nacional de Colombia, Sede Manizales, Colombia. Correo electrónico: anfrojasgo@unal.edu.co.

3 Bióloga, Ph.D. Profesora Asociada en Dedicación Exclusiva. Miembro del Grupo de Investigación en Bacterias Ácido Lácticas y sus Aplicaciones Biotecnológicas Industriales, Facultad de Ingeniería y Arquitectura, Universidad Nacional de Colombia, Sede AA 127. Correo electrónico snrodriguezba@unal.edu.co Fuentes de recursos: Colciencias, programa de Jóvenes Investigadores convocatoria 617 del 2013, Vicerrectoría de Investigación convocatoria del programa nacional de proyectos para el fortalecimiento de la investigación, la creación y la innovación en posgrados de la Universidad Nacional de Colombia 2013-2015 proyecto código 19098.
} 
This a systematic search in several databases on the most widely used probiotics in the food industry, their catalytic capacity in food matrices, encapsulation methods, types of matrices in encapsulation, stability in gastrointestinal conditions and release mechanisms. It was found that encapsulation, besides favoring the stability of probiotic microorganisms against adverse factors, conditions, according to their characteristics, their application and incorporation in food matrices of different qualities.

Key words: functional foods, probiotics, encapsulation matrix, catalytic capacity, probiotic release.

\section{INTRODUCCIÓN}

Gracias al creciente interés de los consumidores por buscar alimentos sanos que favorezcan su bienestar, el mercado de los alimentos con beneficios adicionales ofuncionales ha generado un creciente interés en los últimos años $(1,2,3)$. El término de alimento funcional fue presentado por primera vez en Japón en la década de los 80 cuando se publicó el reglamento de los "Alimentos para uso específico en la salud" (Foods for Specified Health Use o FOSHU). Con ello se hace referencia a los alimentos procesados que contienen ingredientes que cumplen con una función específica y benéfica en el organismo humano, más allá de su contenido nutrimental. El efecto benéfico de estos alimentos se atribuye a los llamados componentes activos $(4,5)$. La actividad fisiológica se puede dar sobre el sistema cardiovascular, gastrointestinal, neuronal y pueden tener efectos antioxidante, anticancerígeno, antidiabético y antiinflamatorios (6). Los microorganismos probióticos son considerados compuestos activos por sus beneficios en la salud del consumidor, como la prevención de diarreas infecciosas, la prevención de diarrea asociada al uso de antibióticos, el tratamiento de la intolerancia a la lactosa, la disminución de niveles de amoniaco en la sangre, la absorción de colesterol y la inhibición de formación de tumores, así como la mejora en la digestibilidad de la lactosa, entre otros $(1,7)$. Sin embargo, los probióticos suelen presentar una alta susceptibilidad frente a tratamientos tecnológicos, almacenamiento y condiciones gastrointestinales. Esta situación hace necesario, en algunos casos, aplicar tecnologías que favorezcan su estabilidad y viabilidad para permanecer fisiológicamente activos al momento del consumo, condición necesaria para considerarse alimento probiótico y conferir al consumidor los beneficios que promete.

La encapsulación es un proceso aplicado para proteger, mediante un material de recubrimiento o material pared, la estabilidad, biodisponibilidad y conservación de los componentes bioactivos y así mismo la viabilidad en microorganismos $(8,9)$. Para la selección de un adecuado material pared se deberán considerar aspectos como grado alimenticio, costos y propiedades físico-químicas como la solubilidad, peso molecular, transición vítrea / fusión, cristalinidad, difusividad, formación de películas y propiedades emulsionantes (10) biodegradabilidad y capacidad para formar una barrera entre el interior de la cápsula y sus alrededores. Se deberán consideraradicionalmente, las propiedades de la matriz alimenticia a la cual se incorporaran los encapsulados (11). Existen numerosas técnicas como la atomización, coacervación compleja, polimerización interfacial e inclusión molecular, liofilización, emulsificación, liposomas y gelificación iónica, que son empleadas para la encapsulación de microorganimos probióticos $(12,13$ 14, 15). La atomización o secado por aspersión es una de las técnicas más ampliamente para este fin, es flexible, segura y económica; por 
Yeimy Alejandra Rodríguez R., Andrés Felipe Rojas G. y Sneyder Rodríguez B.

lo general, se obtienen polvos finos entre 10-100 $\mu \mathrm{m}$ y partículas de mayor tamaño entre 2 y $3 \mathrm{~mm}$ (16). Los microencapsulados son comúnmente distribuidos en polvo o incorporados en matrices alimenticias, en plaguicidas, en fármacos, entre otros; en sus diferentes aplicaciones se deben considerar como criterios de estabilidad la interacción con la matriz, las propiedades fisicoquímicas, propiedades de emulsificación, etc. (2).

Los materiales de recubrimiento más empleados en la encapsulación de bioactivos son los lípidos, piolisacáridos (almidón y sus derivadosamilosa, amilopectina, dextrinas maltodextrinas, celulosa y derivados), exudados y extractos de plantas (goma arábiga, goma tragacanto, goma mesquita, entre otras) carragenatos, alginatos, adicionalmente proteínas como suero lácteo y proteína aislada de soya (15). En términos generales, los polisacáridos presentan una alta facilidad para formar macropartículas esféricas durante el secado, por lo que son ampliamente usados (18).

Este documento tiene como objetivo realizar una breve presentación acerca de la microencapsulación de microorganismos probióticos para su incorporación en alimentos, su estabilidad, actividad en los alimentos y los mecanismos de liberación.

\section{Los probióticos: compuestos fisiológicamente activos}

Los microorganismos probióticos son microorganismos vivos que al ser suministrados en cantidades adecuadas confieren un beneficio a la salud del huésped (5). Los microorganismos deben cumplir con características específicas para que sean considerados como probióticos; algunas características son: resistencia al ácido o a la bilis, adhesión a las células intestinales presentes en los humanos, ser seguros en el momento del consumo, inhibir bacterias patógenas, poder colonizar el intestino y la sustentación de estudios que comprueben los posibles efectos benéficos en la salud (19). Entre los microorganismos probióticos se encuentran principalmente las especies de Lactobacillus y Bifidobacterium, levaduras como Saccharomyces cerevisiae y algunas especies de Bacillus, entre otros $(3,20)$. La atribución de efectos benéficos a las especies probióticas ha generado un creciente interés para su aplicación en alimentos y nutraceúticos $(4,21)$. Se atribuye a ellos la mejora en el equilibrio intestinal, al inhibir mediante diversos mecanismos, el crecimiento de varios grupos microbianos potencialmente patógenos. Esto sucede por la capacidad de algunas especies para interferir con la adhesión de especies patógenas a las superficies de las células intestinales; este efecto es atribuido a la producción de ácidos orgánicos y a la secreción de compuestos antimicrobianos, como las bacteriocinas $(22,23)$. En la tabla 1 se presentan algunos ejemplos de bacterias probióticas y sus productos alimenticios comercializados en el mundo (20).

\section{Actividad catalitica de los probióticos en matrices alimenticias}

Los microorganismos probióticos son adicionados en los alimentos en concentraciones adecuadas $\left(>10^{8}\right)$, con el fin de que el consumidor tras su procesamiento, almacenamiento y distribución reciba los beneficios que ellos le confieren. En algunos casos, la incorporación de los probióticos genera en los alimentos cualidades organolépticas deseables (24). Estudios recientes han demostrado que las cepas potencialmente probióticas pueden actuar como biocatalizadores al realizar trasformaciones que mejoran el alimento durante la elaboración, confiriéndole características organolépticas y nutricionales deseables (25). En el caso de las bacterias ácido lácticas (BAL) probióticas, además de producir ácido láctico por lo cual se conocen como bacterias acidificantes, contribuyen a la formación de sabor, de aroma, de textura y mejoran el valor nutricional de los alimentos fermentados, lo cual contribuye a su vez a la digestibilidad de alimentos y la preservación del 
producto final (24). Las BAL producen pequeñas cantidades de acetaldehídos y diacetilo, los cuales otorgan aromas y sabores agradables a los alimentos, además, algunas BAL presentan actividad lipolítica y proteolítica que influencia la formación de compuestos de aromas y sabores típicos en alimentos fermentados $(24,26)$. Otros ejemplos de biotransformaciones son las causadas por el Bacillus subtilis CS90, que gracias a la actividad catalítica de la $\beta$ - Glucosidasa y la esterasa, mejoran la disponibilidad en el contenido de flavonas, flavonoides e isoflavonas $(27,28)$. Los probióticos también se usan para disminuir el contenido de algunos compuestos no deseados en los alimentos, particularmente, el Lactobacillus fermentum CRL722, es empleado por su alta actividad $\alpha$-galactosidasa para reducir el contenido de lactosa en la leche, causante de flatulencias en intolerantes $(25,29)$.

Tabla 1. Microorganismos probióticos empleados en productos comerciales.

\begin{tabular}{lll}
\hline \multicolumn{1}{c}{ Cepa } & \multicolumn{1}{c}{ Marca } & \multicolumn{1}{c}{ Fabricante } \\
\hline Bifidobacterium animalis subp. Lactis Bb-12 & Activia & Danone/Dannon \\
Bifidobacterium breve Yakult & Bifiene & Yakult \\
Bifidobacterium infantis 35624 & Align & Procter \& Gamble \\
Enterococcus LAB SF 68 & Bioflorin & Cerbios-Pharma \\
Lactobacillus casei DN-114 001 & Actimel, DanActive & Danone/Dannon \\
Lactobacillus johnsonii La1 (Lj1) & LC1 & Nestlé \\
Lactococcus lactis L1A & Norrmejerier & Nestlé \\
Lactobacillus plantarum 299V & GoodBelly, ProViva & NextFoods Probi \\
Lactobacillus rhamnosus ATCC 53013 (LGG) & Vifit y otros & Valio \\
Lactobacillus salivarius UCC118 & Verum & Norrmejerier \\
\hline
\end{tabular}

Los atributos sensoriales de los alimentos también se pueden modificar por la actividad catalítica de los microrganismos probióticos. Estudios como la identificación de compuestos en bebidas de café, han demostrado que algunos de ellos contribuyen al sabor amargo del café tostado modificando sus características organolépticas (30), algunos de estos cambios se deben a la enzima esterasa, la cual cataliza la hidrólisis de los ácidos clorogénicos (CGAs), antioxidante del café en los ácidos quínico y cafeico (31).

Obtención de microorganismos probióticos y alimentos funcionales

Los microorganismos probióticos provienen de fuentes vegetales y animales, en este último caso suelen ser aislados del tracto gastrointestinal, de la saliva y la vagina. Su aislamiento requiere de una adecuada selección de las cepas, identificación mediante técnicas bioquímicas y moleculares, y la evaluación de su inocuidad y beneficios para la salud. Las cepas probióticas deben ser generalmente reconocidas como seguras o GRAS por sus siglas en inglés. Existen varios métodos para la obtención de biomasa de los microorganismos probióticos: entre ellos, la fermentación en el alimento que se convertirá en la matriz portadora final; caso común para los productos lácteos (yogur, queso, mantequilla, etc.), la chucrut, panificados, encurtidos, bebidas, entre otros (32). Otra ruta es aquella en donde el microorganismo es fermentado en un medio de cultivo diferente a la matriz en la que se incorporará; para esto, la fermentación se realiza en medios de cultivo diseñados con un aporte óptimo de las nutrientes exigidos por estos microrganismos, como el caldo comercial MRS formulado por De Man, Rogosa 
Yeimy Alejandra Rodríguez R., Andrés Felipe Rojas G. y Sneyder Rodríguez B.

y Sharpe (33) y recomendado para el cultivo de lactobacillus y otras bacterias lácticas. También se utilizan medios de cultivo diseñados a partir de sustratos naturales como el suero lácteo con o sin suplementación $(75,33)$. Los microorganismos son inoculados e incubados en ambientes generalmente microaerófilos, donde dependiendo de la especie, de las condiciones de fermentación y del medio de cultivo, alcanzarán altas tasas de crecimiento $(\mu$ células $\mathrm{h}^{-1}$ ) (35). Una vez terminada la fermentación, los microorganismos son separados del medio de cultivo, generalmente por centrifugación o filtración y posteriormente lavados. La biomasa obtenida es secada y almacenada hasta su uso, o también puede ser incorporada en fresco sobre la matriz alimenticia final (25). Para la obtención de los alimentos funcionales probióticos se usan tecnologías que involucran la ingeniería de matrices y en ellas la impregnación a vacío, por la cual y gracias a los conocimientos que se tienen sobre la composición, estructura y propiedades del alimentos, es posible la incorporación de microorganismos y otros agentes bioactivos en el interior de la matriz estructural de los alimentos (36). Durante su aplicación, actúa un mecanismo hidrodinámico de trasferencia de masa sólidoporo-líquido; la técnica de impregnación al vacío permite mejorar las características sensoriales y funcionales del alimento $(4,37,38)$. En términos generales, para el desarrollo de alimentos funcionales probióticos, se requiere de elevados costos y de conocimientos complejos, por lo que es necesario tener en cuenta los siguientes pasos (39).

1. Selección y aislamiento de la/las cepa/s probiótica/s.

2. Producción y secado de biomasa.

3. Desarrollo de la matriz alimenticia con la adición de la/s cepa/s.

4. Validación de las propiedades benéficas del alimento.

5. Escalamiento del cultivo probiótico.

6. Escalamiento del alimento con probiótico.

7. Determinación de la vida útil y de la calidad.

\section{Encapsulación de probióticos}

La encapsulación es un método mediante el cual se protege un componente bioactivo de factores medioambientales al ser recubiertos por una matriz protectora. Pueden ser preservados por diferentes técnicas de encapsulación donde se mantienen aislados del entorno hasta su liberación (40). Entre las técnicas de encapsulación utilizadas se encuentran: la emulsión, el secado por aspersión, la coacervación compleja, la gelificación iónica y la liofilización $(15,41,42)$. Muchos autores han investigado en las diversas técnicas de encapsulación los mecanismos adicionales que permiten proteger los microorganismos de condiciones ambientales adversas, por lo que es importante considerar los siguientes factores durante la encapsulación, procesamiento y el almacenamiento $(15,17,41$, $42,43)$.

- Tipo de cepa.

- Tolerancia al estrés.

- El material pared o la solución en la que se va a encapsular.

- La temperatura de secado.

- El tiempo de exposición al calor.

- Los mecanismos oxidativos y la tensión mecánica.

- La temperatura de transición vítrea.

- La actividad del agua $\left(a_{w}\right)$.

- El material de empaque y las condiciones de almacenamiento.

\section{Material pared}

La selección del material pared para la encapsulación de microorganismos probióticos, juega un papel muy importante, pues de este dependerá la eficaz protección contra factores adversos. Para la encapsulación se utilizan diferentes tipos de material pared, como proteínas, lípidos y carbohidratos $(7,8,9,41$, 43, 44, 48, 49). Entre las proteínas, algunos investigadores han trabajado con polipeptonas, proteínas de soya, derivados de leche, gelatinas, entre otros. Entre los lípidos se han aplicado 
liposomas, parafinas, ácido esteárilico y esteárico, entre otros. Los materiales pared a base de carbohidratos pueden ser entre otros almidones, goma arábiga, prebióticos como maltodrextrina, fosfooligosacáridos (FOS) e inulina. El empleo de almidones durante la encapsulación genera una desventaja, puesto que cuando la concentración del material encapsulante es muy alta, se pueden ocasionar problemas durante el secado debido a altas viscosidades (45).

Viabilidad de probióticos bajo condiciones gastrointestinales

Los probióticos deben ser resistentes a condiciones gastrointestinales y ser capaz de tolerar valores de $\mathrm{pH}$ muy bajos. En algunos casos se han modificado genéticamente las cepas, con el fin de potenciar su resistencia a la alta acidez, como es el caso de las sales biliares (44). La viabilidad de los probióticos depende también del material en el que se encapsule, durante los métodos de obtención de las cápsulas se debe de elegir muy bien el material portador que garantice una alta estabilidad de los microorganismos en el tracto gastrointestinal (47). Cuando las células se encuentran encapsuladas, presentan una mayor resistencia a los jugos gástricos que cuando se encuentran en estado libre. Se han usado materiales portadores como el alginato para mejorar la supervivencia de algunas Bifidobacterium (48). También se han encapsulado probióticos como Bifidobacterium adolesensis en alginatos con mezclas de biopolímeros (49), Lactobacillus acidophilus y Lactobacillus rhamnosus PTCC1643 PTCC1637 en perlas de alginato de calcio con y sin recubrimiento de alginato de sodio, en donde los autores encontraron que las perlas recubiertas de alginato podrían reducir la pérdida de viabilidad de las cepas ante las condiciones simuladas gastrointestinales de pH 1,5 (50).

\section{Mecanismos de liberación de las microcápsulas}

Los microorganismos probióticos deben superar los problemas tecnológicos, y presentar alta capacidad de resistencia a ácidos en el medio gástrico y a las soluciones biliares, para posteriormente ser liberadas en el tracto gastrointestinal (51). Los componentes activos microencapsulados liberan su contenido a velocidades controladas durante prolongados periodos de tiempo, esta liberación puede ocurrir de diferentes formas; como la fusión o disolución en donde las cápsulas son descompuestas por la adición de un solvente o por la acción de calor, aumentando la humedad cuando el recubrimiento es hidrosoluble (52). Otro mecanismo de liberación es la presión física, en donde las cápsulas son liberadas por fuerzas externas o por fricción (47). La difusión es un método de liberación en donde las cápsulas salen de su matriz guiadas por gradientes de concentración, fuerzas atractivas de enlace como puentes de hidrógeno, fuerzas de Van der Waals, grado de entrecruzamiento y cristalinidad (52), el mecanismo de difusión está controlado por la solubilidad y la permeabilidad del agente encapsulado en el material protector.

\section{PERSPECTIVAS DE LA ENCAPSULACIÓN DE PROBIÓTICOS}

El mercado de los alimentos probióticos representa el mayor renglón de ingresos en área de los alimentos funcionales, pues los consumidores son cada vez más conscientes de los beneficios de la ingesta de alimentos con microorganismos saludables. Tradicionalmente, los alimentos lácteos han sido empleados como matrices para los microorganismos probióticos gracias a su habilidad para crecer en este sustrato. La búsqueda de diversidad e innovación de nuevos productos ha llevado a la industria alimentaria a investigar en formas de favorecer la viabilidad de los probióticos frente a factores adversos que reducen su estabilidad durante el procesamiento, almacenamiento y consumo. En este caso, la encapsulación se ha convertido en la alternativa más viable para incorporar microorganismos en matrices que 
Yeimy Alejandra Rodríguez R., Andrés Felipe Rojas G. y Sneyder Rodríguez B.

finalmente servirán como vehículos de bacterias probióticas. Entre estas nuevas matrices se tienen productos vegetales, carnes maduradas, chocolates, harinas instantáneas, coberturas y rellenos de golosinas.

Finalmente, y tras la adecuada selección del método de encapsulación, del material pared y de la matriz alimenticia que permitan su viabilidad, en un número minino según la legislación $\left(\geq 10^{6} \mathrm{UFCg}^{-1} \mathrm{o} \mathrm{ml}^{-1}\right)$, serán sus características organolépticas y los atributos para la salud los que determinen la aceptación de los alimentos funcionales por parte del consumidor.

\section{CONCLUSIONES}

Con la demanda de alimentos saludables por parte del consumidor, es creciente el mercado de alimentos funcionales probióticos. Ello exige la búsqueda de nuevas matrices alimenticias que sirvan de vehículo a los microorganismos probióticos y con ello la selección adecuada de la técnica de encapsulación y del material pared que favorezcan la estabilidad y la viabilidad del probiótico durante el procesamiento, almacenamiento y consumo. Finalmente, es importante el desarrollo de investigaciones que den soporte a los atributos para la salud que se le confieren al microorganismo probiótico agregado.

\section{AGRADECIMIENTOS}

Los autores agradecen a Colciencias y a la Universidad Nacional de Colombia por el apoyo económico a través del programa de Jóvenes Investigadores e Innovadores convocatoria 617 del 2013.

A la Vicerrectoría de Investigación por su apoyo a través de la convocatoria del Programa Nacional de Proyectos para el Fortalecimiento de la Investigación, la Creación y la Innovación en Posgrados de la Universidad Nacional de Colombia 2013-2015 proyecto código 19098.

\section{REFERENCIAS}

1. Kailasapathy K. Encapsulation technologies for functional foods and nutraceutical product development. Perspectives in Agriculture, Veterinary Science, Nutrition and Natural Resources 2009; 4:1-19.

2. González-Aguilar EG, García EÁ, Los alimentos funcionales. Revista fitotecnia mexicana 2015; 38 : 233.

3. Jiménez-Colmenero F. Multiple emulsions; bioactive compounds and functional foods. Nutrición hospitalaria 2013; 28:1413-1421.

4. Cortés M, Puente L. Alimentos funcionales: una historia con mucho presente y futuro. Vitae 2005; 12:5-14.

5. FAO/WHO (Food and Agriculture Organization/World Health Organization). Working Group Report on Drafting Guidelines for the Evaluation of Probiotics in Food. Guidelines for the Evaluation of Probiotics in Foods. London, Ontario, Canada: s.n.; 2002. p. 1-11.

6. Ignat I, Volf I, Popa VI. A critical review of methods for characterisation of polyphenolic compounds in fruits and vegetables. Food Chemistry $2011 ; 126: 1821-1835$.

7. Capela P, Hay TK, Shah NP. Effect of cryoprotectants, prebiotics and microencapsulation on survival of probiotic organisms in yoghurt and freeze-dried yoghurt. Food Research International 2006; 39:203-211. 
8. Fritzen-Freire CB, Prudêncio ES, Amboni RD, Pinto SS, Negrão-Murakami AN, Murakami FS. Microencapsulation of bifidobacteria by spray drying in the presence of prebiotics. Food Research International 2012; 45: 06-312.

9. Khem S, Small DM, May BK. The behaviour of whey protein isolate in protecting Lactobacillus plantarum. Food chemistry 2016; 190:717-723.

10. Gharsallaoui A, Roudaut G, Chambin O, Voilley A, Saurel R. Applications of spray-drying in microencapsulation of food ingredients: An overview. Food Research International 2007; 40:1107-1121

11. Stoll L, Costa TM, Jablonski A, Flôres S, de Oliveira Rios A. Microencapsulation of anthocyanins with different wall materials and its application in active biodegradable films. Food and Bioprocess Technology 2015; 8:1-10.

12. Li Y, Ai L, Yokoyama W. Properties of chitosan-microencapsulated orange oil prepared by spray-drying and its stability to detergents. Journal of Agricultural and Food Chemistry 2013; 61:3311-3319.

13. Eratte D, McKnight S, Gengenbach TR, Dowling K, Barrow CJ, Adhikari BP. Co-encapsulation and characterisation of omega-3 fatty acids and probiotic bacteria in whey protein isolate-gum Arabic complex coacervates. Journal of Functional Foods 2015; 19:882-892.

14. Xu M, Gagné-Bourque F, Dumont M-J, Jabaji S. Encapsulation of Lactobacillus casei ATCC 393 cells and evaluation of their survival after freeze-drying, storage and under gastrointestinal conditions. Journal of Food Engineering 2016; 168:52-59.

15. Da Silva TM, Rodrigues LZ, Codevilla CF, da Silva CD, de Menezes CR. Coacervação complexa: uma técnica para a encapsulação de probióticos. Ciencia \& Natura 2016; 37:49-55.

16. Pinto SS, Fritzen-Freire $C B$, Benedetti $S$ et al. Potential use of whey concentrate and prebiotics as carrier agents to protect Bifidobacterium-BB-12 microencapsulated by spray drying. Food Research International 2015; 67:400-408.

17. Chávez BE, Ledeboer a. M. Drying of Probiotics: Optimization of Formulation and Process to Enhance Storage Survival. Drying Technology 2007; 25:1193-1201.

18. Burgain J, Gaiani C, Linder M, Scher J. Encapsulation of probiotic living cells: From laboratory scale to industrial applications. Journal of Food Engineering2011; 104:467-483.

19. Saarela M, Mogensen G, Fonde R. Probiotic bacteria: safety, functional and technological properties. Journal of Biotechnology 2000; 84:197-215.

20. WGO (World Gastroenterology Organisation) Practice Guideline: Probiotics and prebiotics, 2011.

21. Picard C, Fioramonti J, Francois A, Robinson T, Neant F, Matuchansky C. Bifidobacteria as probiotic agents physiological effects and clinical benefits. Alimentary Pharmacology \& Therapeutics 2005; 22:495-512.

22. Bogsan CS, Ferreira L, Maldonado C, Perdigon G, Almeida SR, Oliveira MN. Fermented or unfermented milk using Bifidobacterium animalis subsp. lactis HN019: Technological approach determines the probiotic modulation of mucosal cellular immunity. Food Research International 2014; 64:283-288.

23. Liu G, Ren L, Song Z, Wang C, Sun B. Purification and characteristics of bifidocin A, a novel bacteriocin produced by Bifidobacterium animals BB04 from centenarians' intestine. Food Control 2015; 50:889895.

24. Parra RA. Bacterias ácido lácticas: papel funcional en los alimentos. Facultad de Ciencias Agropecuarias 2010; 8:93-105.

25. Makinen K, Berger B, Bel-Rhlid R, Ananta E. Science and technology for the mastership of probiotic applications in food products. Journal of Biotechnology 2012; 162:356-365.

26. Ojha KS, Kerry JP, Duffy G, Beresford T, Tiwari BK. Technological advances for enhancing quality and safety of fermented meat products. Trends in Food Science \& Technology 2015; 44:105-116.

27. Ding WK, Shah NP. Enhancing the biotransformation of isoflavones in soymilk supplemented with lactose using probiotic bacteria during extended fermentation. Journal of Food Science 2010; 75:M140-9. 
Yeimy Alejandra Rodríguez R., Andrés Felipe Rojas G. y Sneyder Rodríguez B.

28. Cho KM, Lee JH, Yun HD, Ahn BY, Kim H, Seo WT. Changes of phytochemical constituents (isoflavones, flavanols, and phenolic acids) during cheonggukjang soybeans fermentation using potential probiotics Bacillus subtilis CS90. Journal of Food Composition and Analysis 2011; 24:402-410.

29. LeBlanc JG, Piard J-C, Sesma F, de Giori GS. Lactobacillus fermentum CRL 722 is able to deliver active alpha-galactosidase activity in the small intestine of rats. FEMS Microbiology Letters 2005; 248:177-182.

30. Frank O, Zehentbauer G, Hofmann T. Bioresponse-guided decomposition of roast coffee beverage and identification of key bitter taste compounds. European Food Research and Technology 2005; 222:492-508.

31. Scherbl D, Muentnich S, Richling E. In vitro absorption studies of chlorogenic acids from coffee using the Ussing chamber model. Food Research International2014; 63:456-463.

32. Marafon AP, Sumi A, Alcântara MR, Tamime AY, Nogueira de Oliveira M. Optimization of the rheological properties of probiotic yoghurts supplemented with milk proteins. LWT - Food Science and Technology 2011; 44:511-519.

33. De Man, JC., Rogosa, D., Sharpe M. A medium for the cultivation of lactobacilli. Journal of Applied Bacteriology 1960; 23:130-135.

34. Urribarrí L, Vielma A, Paéz G, Ferrer J, Mármol Z. Producción de ácido láctico a partir de suero de leche, utilizando Lactobacillus helveticus en cultivo continuo. Revista Científica 2004; 14:297-302.

35. Garro MS, de Valdez GF, de Giori GS. Temperature effect on the biological activity of Bifidobacterium longum CRL 849 and Lactobacillus fermentum CRL 251 in pure and mixed cultures grown in soymilk. Food Microbiology 2004; 21:511-518.

36. Rodríguez-Barona S, Giraldo GI, Zuluaga YP. Evaluación de la incorporación de fibra prebiótica sobre la viabilidad de Lactobacillus casei impregnado en matrices de mora (Rubus glaucus). Información tecnológica 2015; 26:25-34.

37. Restrepo AM, Cortés M, Márquez CJ. Uchuvas (Physalis peruviana I) mínimamente procesadas fortificadas con vitamina E. Vitae 2009; 16:19-30.

38. Arango ZT, Cortés M, Montoya OI. Frutos de uchuva (physalis peruviana I.) ecotipo "Colombia" mínimamente procesados, adicionados con microorganismos probióticos utilizando la ingeniería de matrices. Revista Facultad Nacional de Agronomía 2010; 63:5395-5407.

39. Parzanese TM. Tecnologías para la Industria Alimentaria: Desarrollo de prebióticos y probióticos. Alimentos Argentinos Ficha 12. 2014; 1-9.

40. López A, Deladino L, Navarro A, Martino M. Encapsulación de compuestos bioactivos con alginatos para la industria de alimentos. @limentech Ciencia y Tecnología Alimentaria 2012; 10:18-27.

41. Heidebach $T$, Först $P$, Kulozik U. Influence of casein-based microencapsulation on freeze-drying and storage of probiotic cells. Journal of Food Engineering 2010; 98:309-316.

42. Valero-Cases $\mathrm{E}$, Frutos MJ. Effect of different types of encapsulation on the survival of Lactobacillus plantarum during storage with inulin and in vitro digestion. LWT - Food Science and Technology 2015; 64:824-828.

43. Tripathi MK, Giri SK. Probiotic functional foods: Survival of probiotics during processing and storage. Journal of Functional Foods 2014; 9:225-241.

44. Lopera S, Guzmán C, Cataño C. Desarrollo y caracterización de micropartículas de ácido fólico formadas por secado por aspersión, utilizando goma arábiga y maltodextrina como materiales de pared. Vitae 2009; 16:55-65.

45. Martin MJ, Lara-Villoslada F, Ruiz M, Morales ME. Effect of unmodified starch on viability of alginateencapsulated Lactobacillus fermentum CECT5716. LWT. Food Science and Technology2013; 53:480486. 
46. Turpin W, Humblot C, Guyot JP. Genetic screening of functional properties of lactic acid bacteria in a fermented pearl millet slurry and in the metagenome of fermented starchy foods. Applied and Environmental Microbiology2011; 77:8722-8734.

47. Pérez-Leonard H, Bueno-García G, Brizuela-Herrada MA. Microencapsulación: una vía de protección para microorganismos probióticos. ICIDCA. Sobre los Derivados de la Caña de Azúcar 2013; 47:1425.

48. Annan NT, Borza a. D, Hansen LT. Encapsulation in alginate-coated gelatin microspheres improves survival of the probiotic Bifidobacterium adolescentis 15703T during exposure to simulated gastrointestinal conditions. Food Research International 2008; 41:184-193.

49. Khan NH, Korber DR, Low NH, Nickerson MT. Development of extrusion-based legume protein isolatealginate capsules for the protection and delivery of the acid sensitive probiotic, Bifidobacterium adolescentis. Food Research International 2013; 54: 730-737.

50. Mokarram RR, Mortazavi S, Najafi MB, Shahidi F. The influence of multi stage alginate coating on survivability of potential probiotic bacteria in simulated gastric and intestinal juice. Food Research International 2009; 42:1040-1045.

51. Champagne CP, Fustier P. Microencapsulation for the improved delivery of bioactive compounds into foods. Current Opinion in Biotechnology 2007; 18:184-190.

52. Aldana AS, Sandoval ER, Aponte AA. Encapsulación de Aditivos para la Industria de Alimentos. Ingenieria y Competitividad 2004; 5:73-83. 